\title{
Reconstructing integer sets from their representation functions
}

\author{
Vsevolod F. Lev \\ Department of Mathematics \\ University of Haifa at Oranim \\ Tivon 36006, Israel \\ seva@math.haifa.ac.il
}

Submitted: Oct 5, 2004; Accepted: Oct 27, 2004; Published: Nov 3, 2004

Mathematics Subject Classifications: 11B34, 05A17, 11B13

\begin{abstract}
We give a simple common proof to recent results by Dombi and by Chen and Wang concerning the number of representations of an integer in the form $a_{1}+a_{2}$, where $a_{1}$ and $a_{2}$ are elements of a given infinite set of integers. Considering the similar problem for differences, we show that there exists a partition $\mathbb{N}=\cup_{k=1}^{\infty} A_{k}$ of the set of positive integers such that each $A_{k}$ is a perfect difference set (meaning that any non-zero integer has a unique representation as $a_{1}-a_{2}$ with $a_{1}, a_{2} \in A_{k}$ ). A number of open problems are presented.
\end{abstract}

\section{Introduction and summary}

For a set $A \subseteq \mathbb{Z}$ and an integer $n \in \mathbb{Z}$ consider the representation functions

$$
\begin{aligned}
& R_{A}^{(1)}(n)=\left\{\left(a_{1}, a_{2}\right) \in A \times A: a_{1}+a_{2}=n\right\}, \\
& R_{A}^{(2)}(n)=\left\{\left(a_{1}, a_{2}\right) \in A \times A: a_{1}+a_{2}=n, a_{1}<a_{2}\right\},
\end{aligned}
$$

and

$$
R_{A}^{(3)}(n)=\left\{\left(a_{1}, a_{2}\right) \in A \times A: a_{1}+a_{2}=n, a_{1} \leq a_{2}\right\} .
$$

To what extent do $R_{A}^{(j)}(n)$ determine the set $A$ ? Problems of this sort were, to our knowledge, first studied by Nathanson in [N78]. Let $\mathbb{N}$ denote the set of all positive integers. In his research talks and private communications, Sárközy has raised the following question: do there exist $A, B \subseteq \mathbb{N}$ with the infinite symmetric difference such that $R_{A}^{(j)}(n)=R_{B}^{(j)}(n)$ for all, but finitely many $n \in \mathbb{N}$ ?

Dombi noticed in [D02] that the answer is negative for $j=1$, by the simple observation that $R_{A}^{(1)}(n)$ is odd if and only if $n=2 a$ for some $a \in A$. On the other hand, he has shown that for $j=2$ the answer is positive and indeed, there is a partition $\mathbb{N}=A \cup B$ such that $R_{A}^{(2)}(n)=R_{B}^{(2)}(n)$ for all $n \in \mathbb{N}$. 
Theorem 1 (Dombi [D02]) Define the mapping $T: \mathbb{N} \rightarrow\{1,-1\}$ by

$$
T(1)=1, \quad T(2 n)=-T(2 n-1), T(2 n+1)=T(n+1) ; \quad n \in \mathbb{N}
$$

and let

$$
A=\{n \in \mathbb{N}: T(n)=1\}, B=\{n \in \mathbb{N}: T(n)=-1\} .
$$

Then $R_{A}^{(2)}(n)=R_{B}^{(2)}(n)$ for all $n \in \mathbb{N}$.

For the function $R_{A}^{(3)}(n)$ the problem was solved by Chen and Wang in [CW03].

Theorem 2 (Chen and Wang [CW03]) ${ }^{1}$ Define the mapping $T: \mathbb{N} \rightarrow\{1,-1\}$ by

$$
T(1)=1, T(2 n)=-T(2 n-1), T(2 n+1)=-T(n+1) ; \quad n \in \mathbb{N}
$$

and let

$$
A=\{n \in \mathbb{N}: T(n)=1\}, B=\{n \in \mathbb{N}: T(n)=-1\} .
$$

Then $R_{A}^{(3)}(n)=R_{B}^{(3)}(n)$ for all integer $n \geq 3$.

Below we give Theorems 1 and 2 a new simple proof, establishing both results through one common argument which also shows that the constructions of Dombi and ChenWang are, essentially, unique. We then proceed to investigate the parallel problem for differences.

Let $r_{A}(n)$ denote the number of representations of the integer $n$ as a difference of two elements of the set $A \subseteq \mathbb{Z}$ :

$$
r_{A}(n)=\left\{\left(a^{\prime}, a^{\prime \prime}\right) \in A \times A: a^{\prime \prime}-a^{\prime}=n\right\} .
$$

It is not difficult to see that for any finite partition of $\mathbb{N}$ one can find a partition set, say $A$, such that there are arbitrarily large integer $n$ with $r_{A}(n)=\infty$. On the other hand, we were able to partition $\mathbb{N}$ into the infinite number of subsets with identical finite difference representation functions. Indeed, our subsets are perfect difference sets. (Recall, that $A \subseteq \mathbb{Z}$ is a perfect difference set if any non-zero integer has a unique representation as a difference of two elements of $A$; in our terms, $r_{A}(n)=1$ for any $n \in \mathbb{N}$.) Moreover, one can arrange it so that the subsets in question have completely different structure.

Theorem 3 There is a partition $\mathbb{N}=\cup_{k=1}^{\infty} A_{k}$ of the set of all positive integers such that each $A_{k}$ is a perfect difference set and $\left|A_{i} \cap\left(A_{j}+z\right)\right| \leq 2$ for any $i, j, z \in \mathbb{N}$.

\footnotetext{
${ }^{1}$ The way we present Theorems 1 and 2 emphasizes the striking similarity between the partitions $\mathbb{N}=A \cup B$ considered in these theorems. Ironically, Dombi conjectured that sets $A, B \subseteq \mathbb{N}$ with the infinite symmetric difference satisfying $R_{A}^{(3)}(n)=R_{B}^{(3)}(n)$ (for $n$ large enough) do not exist. The result of Chen and Wang shows, however, that such sets do exist and can be obtained by a very minor modification of Dombi's original construction.
} 


\section{The proofs}

Proof of Theorems 1 and 2. Let $A_{2}, B_{2}$, and $T_{2}$ denote the two sets and the mapping of Theorem 1 , and let $A_{3}, B_{3}$, and $T_{3}$ denote the two sets and the mapping of Theorem 2 . For $j \in\{2,3\}$ define

$$
\alpha_{j}(x)=\sum_{a \in A_{j}} x^{a}, \beta_{j}(x)=\sum_{b \in B_{j}} x^{b}, \text { and } \tau_{j}(x)=\sum_{n \in \mathbb{N}} T_{j}(n) x^{n} .
$$

Thus $\alpha_{j}(x), \beta_{j}(x)$, and $\tau_{j}(x)$ converge absolutely for $|x|<1$ and satisfy

$$
\alpha_{j}(x)+\beta_{j}(x)=\frac{x}{1-x}, \alpha_{j}(x)-\beta_{j}(x)=\tau_{j}(x) .
$$

Moreover, it is easily seen that

$$
2 \sum_{n \in \mathbb{N}} R_{A}^{(j)}(n) x^{n}=\left(\alpha_{j}(x)\right)^{2}+(-1)^{j+1} \alpha_{j}\left(x^{2}\right)
$$

and similar identity holds with $B$ and $\beta$ substituted for $A$ and $\alpha$, respectively. Taking into account that the sum $T_{j}(1)+\cdots+T_{j}(n-1)$ vanishes for $n$ odd and equals $-T_{j}(n)$ for $n$ even, we derive from (1) and (2) that

$$
\begin{aligned}
2 \sum_{n \in \mathbb{N}}\left(R_{A}^{(j)}(n)-R_{B}^{(j)}(n)\right) x^{n} & =\left(\left(\alpha_{j}(x)\right)^{2}-\left(\beta_{j}(x)\right)^{2}\right)+(-1)^{j+1}\left(\alpha_{j}\left(x^{2}\right)-\beta_{j}\left(x^{2}\right)\right) \\
& =\frac{x}{1-x} \tau_{j}(x)+(-1)^{j+1} \tau_{j}\left(x^{2}\right) \\
& =\sum_{n \in \mathbb{N}}\left(\sum_{1 \leq i \leq n-1} T_{j}(i)\right) x^{n}+(-1)^{j+1} \sum_{n \in \mathbb{N}} T_{j}(n) x^{2 n} \\
& =\sum_{n \in \mathbb{N}}\left(-T_{j}(2 n)+(-1)^{j+1} T_{j}(n)\right) x^{2 n}
\end{aligned}
$$

for $|x|<1$ and $j \in\{2,3\}$. It remains to observe that $T_{j}(2 n)=(-1)^{j+1} T_{j}(n)$, except if $j=3$ and $n=1$.

Suppose that $\mathbb{N}=A \cup B$ is a partition of the set of positive integers and let $T(n)=1$ if $n \in A$ and $T(n)=-1$ if $n \in B$. Our proof of Theorems 1 and 2 shows that then $R_{A}^{(j)}(n)=R_{B}^{(j)}(n)$ for all sufficiently large $n$ if and only if $T(1)+\cdots+T(2 n)=0$ and $T(2 n)=(-1)^{j+1} T(n)$, for all but finitely many $n \in \mathbb{N}$. The reader will easily check that this is equivalent to the assertion that there exists $n_{0} \in \mathbb{N}$ such that $T(2 n)=-T(2 n-1)$ and $T(2 n-1)=(-1)^{j} T(n)$ for $n \geq n_{0}$, and $T(1)+\cdots+T\left(2 n_{0}\right)=0$. That is, any partition $\mathbb{N}=A \cup B$ satisfying $R_{A}^{(j)}(n)=R_{B}^{(j)}(n)$ for all sufficiently large $n$ is obtained essentially as in Theorems 1 and 2 .

Proof of Theorem 3. Fix a function $f: \mathbb{N} \rightarrow \mathbb{N}$ satisfying

$$
f(2 m-1) \leq m, f(2 m)=m+1 ; \quad m=1,2, \ldots
$$


and such that for any $k \in \mathbb{N}$ the inverse image $f^{-1}(k)=\{n \in \mathbb{N}: k=f(n)\}$ is infinite. Set $A_{k}=\varnothing$ for all $k \in \mathbb{N}$. Our construction involves infinitely many steps which we enumerate by positive integers. At the $n$th step we add one or two elements to the set $A_{f(n)}$ so that (i) every positive integer is added to some $A_{k}$ at certain step; (ii) no positive integer is added to several distinct $A_{k}$ at different steps; (iii) for any $d, k \in \mathbb{N}$ there is a step such that the element(s) added at this step to $A_{k}$ produce(s) a pair $\left(a_{1}, a_{2}\right) \in A_{k} \times A_{k}$ with $a_{2}-a_{1}=d$; (iv) the element(s) added to $A_{k}$ at any step produce(s) no non-trivial equality of the form $a_{1}-a_{2}=a_{3}-a_{4}$ with $a_{1}, a_{2}, a_{3}, a_{4} \in A_{k}$; (v) the element(s) added to $A_{k}$ at any step produce(s) no triple $\left(a_{1}, a_{2}, a_{3}\right) \in A_{k} \times A_{k} \times A_{k}$ which is a shift of another triple $\left(b_{1}, b_{2}, b_{3}\right) \in A_{l} \times A_{l} \times A_{l}$ with some $l \neq k$. Once we manage to satisfy (i)-(v), our proof is over; we now proceed to describe exactly how the elements to be added to $A_{f(n)}$ at the $n$th step are chosen.

If $n=2 m$ is even then it follows from (3) that the set $A_{f(n)}=A_{m+1}$ was not affected by steps $1, \ldots, n-1$. This set, therefore, remains empty by the beginning of the $n$th step, and we initialize it inserting to it the smallest positive integer not contained in $\cup_{l=1}^{m} A_{l}$.

Suppose now that $n$ is odd and write for brevity $k=f(n)$. Let $d$ be the smallest positive integer, not representable as $a_{1}-a_{2}$ with $a_{1}, a_{2} \in A_{k}$. We insert to $A_{k}$ two numbers $z$ and $z+d$, where $z$ is to satisfy the following conditions:

(a) $\{z, z+d\} \cap\left(\cup_{l=1}^{\infty} A_{l}\right)=\varnothing$;

(b) equality $a_{1}-a_{2}=a_{3}-a_{4}$ with $a_{1}, a_{2}, a_{3}, a_{4} \in A_{k} \cup\{z, z+d\}$ holds only trivially; that is, if and only if either $a_{1}=a_{3}$ and $a_{2}=a_{4}$, or $a_{1}=a_{2}$ and $a_{3}=a_{4}$;

(c) none of the triples $\left(a_{1}, z, z+d\right),\left(a_{1}, a_{2}, z\right),\left(a_{1}, a_{2}, z+d\right)$ with $a_{1}, a_{2} \in A_{k}$ are translates of a triple $\left(b_{1}, b_{2}, b_{3}\right)$ with $b_{1}, b_{2}, b_{3} \in A_{l}, l \neq k$.

Clearly, condition (a) excludes only a finite number of possible values of $z$, and a little meditation shows that this is the case also with condition (b). Concentrating on condition (c), we notice that the actual number of values of $l$ to be taken into account is finite, as all but $(n+1) / 2$ sets $A_{l}$ are empty by the beginning of the $n$th step. Furthermore, for any fixed $l$ the number of triples $\left(b_{1}, b_{2}, b_{3}\right)$ with $b_{1}, b_{2}, b_{3} \in A_{l}$ is finite, and the number of possible values of $a_{1} \in A_{k}$ is finite, too. It follows that condition (c) also excludes only finite number of $z$. Thus choosing $z$ is always possible, and this concludes the proof.

\section{Open problems}

We list below some related problems.

The proof of Theorem 3 can be simplified if we wish to construct just one perfect difference set $A \subseteq \mathbb{N}$. In this case we can start with the empty set $A^{(0)}=\varnothing$ and define at the $n$th step $A^{(n)}=A^{(n-1)} \cup\left\{z_{n}, z_{n}+d_{n}\right\}$, where $d_{n}$ is the smallest non-negative integer not representable as $a_{1}-a_{2}$ with $a_{1}, a_{2} \in A^{(n-1)}$, and $z_{n}$ is to be so chosen that $z_{n}, z_{n}+d_{n} \notin A^{(n-1)}$, and no non-trivial equality $a_{1}-a_{2}=a_{3}-a_{4}$ with $a_{1}, a_{2}, a_{3}, a_{4} \in$ 
$A^{(n-1)} \cup\left\{z_{n}, z_{n}+d_{n}\right\}$ is created. The number of choices of $z_{n}$ excluded by these conditions is $O\left(n^{3}\right)$ and since $d_{n}=O\left(n^{2}\right)$, the $n$th element of the resulting set $A$ is $O\left(n^{3}\right)$. It follows that the counting function $A(x)=|A \cap[1, x]|$ satisfies $A(x) \gg x^{1 / 3}$. On the other hand, it is easily seen that for any perfect difference set $A \subseteq \mathbb{N}$ we have $A(x) \ll x^{1 / 2}$.

Problem 1 Does there exist a perfect difference set $A \subseteq \mathbb{N}$ with the counting function $A(x) \gg x^{1 / 2}$ ? If not, is it true that for any $\varepsilon>0$ there exists a perfect difference set $A \subseteq \mathbb{N}$ with $A(x) \gg x^{1 / 2-\varepsilon}$ ? If not, how large can $\liminf \operatorname{in}_{x \rightarrow \infty} \ln A(x) / \ln x$ for a perfect difference set $A \subseteq \mathbb{N}$ be?

The definition of $R_{A}^{(1)}(n)$ extends readily onto the case where $A$ is a subset of an arbitrary abelian group $G$ and $n$ is an element of the group. Suppose that $G$ is finite and for a group character $\chi$ let $\widehat{A}(\chi)=|G|^{-1} \sum_{a \in A} \bar{\chi}(a)$, the Fourier coefficient of the indicator function of $A$. The identity $R_{A}^{(1)}=R_{B}^{(1)}$ translates easily into the requirement that either $\widehat{A}(\chi)=\widehat{B}(\chi)$ or $\widehat{A}(\chi)=-\widehat{B}(\chi)$ hold for any character $\chi$. Though this seems to be a rather strong condition, numerical computations show that for certain groups pairs $(A, B)$ such that $R_{A}^{(1)}=R_{B}^{(1)}$ are not that rare as one could expect. Quite likely, these pairs are not limited to simple special cases as for instance $|A|=|G| / 2, B=G \backslash A$, or $B=\{a+d: a \in A\}$ with a fixed element $d \in G$ of order two. Nevertheless we state our next problem in the most general form.

Problem 2 For any finite abelian group $G$ determine all pairs of subsets $A, B \subseteq G$ such that $R_{A}^{(1)}=R_{B}^{(1)}$.

We note that if $G$ is of odd order then no non-trivial pairs exist, as in this case the values of $n$ for which $R_{A}^{(1)}(n)$ is odd determine the set $A$ uniquely. On the other hand, if $G$ is an elementary 2-group then any two perfect difference sets $A, B \subseteq G$ satisfy $R_{A}^{(1)}=R_{B}^{(1)}$.

As a common generalization of the representation functions $R_{A}^{(1)}$ and $r_{A}$, one can consider two potentially different sets $A, B \subseteq \mathbb{Z}$ and for $n \in \mathbb{Z}$ define

$$
r_{A, B}(n)=\#\{(a, b) \in A \times B: a+b=n\} .
$$

An unpublished observation due to Freiman, Yudin, and the present author is as follows. Suppose that $A$ and $B$ are finite and non-empty, and for $k \in \mathbb{N}$ let $\nu_{k}$ denote the $k$ th largest value attained by $r_{A, B}$. Thus $\left\{\nu_{k}\right\}$ is the spectrum of the function $r_{A, B}$ and we have $\nu_{1} \geq \nu_{2} \geq \cdots, \nu_{1}+\nu_{2}+\cdots=|A||B|$, and $\nu_{k}=0$ for all $k$ large enough. Then

$$
\nu_{k}^{2} \leq \nu_{k}+\nu_{k+1}+\nu_{k+2}+\cdots
$$

for any $k \in \mathbb{N}$.

For the proof we write $A=\left\{a_{1}, \ldots, a_{l}\right\}$ and $B=\left\{b_{1}, \ldots, b_{m}\right\}$ where the elements are numbered in the increasing order, and notice first that

$$
r_{A, B}\left(a_{i}+b_{j}\right) \leq \min \{i+j-1, l+m-(i+j-1)\} ; \quad 1 \leq i \leq l, 1 \leq j \leq m .
$$

For if $a_{i}+b_{j}=a_{u}+b_{v}$ then either $u \leq i$, or $v \leq j$; since there are at most $i$ such representations with $u \leq i$ and at most $j$ representations with $v \leq j$, and one representation 
satisfies both $u \leq i$ and $v \leq j$, we conclude that $r_{A, B}\left(a_{i}+b_{j}\right) \leq i+j-1$. The proof of the estimate $r_{A, B}\left(a_{i}+b_{j}\right) \leq l+m-(i+j-1)$ is almost identical; just notice that if $a_{i}+b_{j}=a_{u}+b_{v}$ then either $u \geq i$ or $v \geq j$. Now we have

$$
\begin{aligned}
\nu_{1}+\cdots+\nu_{k} & \leq \#\left\{(i, j): r_{A, B}\left(a_{i}+b_{j}\right) \geq \nu_{k}\right\} \\
& \leq \#\left\{(i, j): \min \{i+j-1, l+m-(i+j-1)\} \geq \nu_{k}\right\} \\
& =\#\left\{(i, j): \nu_{k} \leq i+j-1 \leq l+m-\nu_{k}\right\} \\
& =\operatorname{lm}-2 \#\left\{(i, j): i+j \leq \nu_{k}\right\} \\
& =l m-\nu_{k}\left(\nu_{k}-1\right)
\end{aligned}
$$

and (4) follows from $l m=\nu_{1}+\cdots+\nu_{k}+\nu_{k+1}+\cdots$.

Problem 3 What are the general properties shared by the functions $r_{A, B}(n)$ (for all finite non-empty $A, B \subseteq \mathbb{Z}$ ), other than that reflected by (4)?

Since the spectrum $\left\{\nu_{k}\right\}$ defines a partition of the integer $|A \| B|$, it can be visualized with a Ferrers diagram corresponding to this partition; that is, an arrangement of $|A||B|$ square boxes in bottom-aligned columns such that the leftmost column is of height $\nu_{1}$, the next column is of height $\nu_{2}$, and so on. For any $t \in \mathbb{N}$, the length of the $t$ th row of this diagram (counting the rows from the bottom) is then $N_{t}=\#\left\{n: r_{A, B}(n) \geq t\right\}$. We notice that from a well-known result of Pollard [P75] it follows that $N_{1}+\cdots+N_{t} \geq t(|A|+|B|-t)$ for any $t \leq \min \{|A|,|B|\}$; one can derive this inequality as a corollary of (4), too.

We conclude our note with two problems due to Gowers and Konyagin, presented here from their kind permission. Both problems pertain to the group $\mathbb{Z} / p \mathbb{Z}$ of residue classes modulo a prime $p$.

Problem 4 (Gowers, personal communication) For a prime $p$, let $A \subseteq \mathbb{Z} / p \mathbb{Z}$ be a subset of cardinality $|A|=(p+1) / 2$. The average value of $R_{A}^{(1)}$ is then $(p+1)^{2} /(4 p)=$ $p / 4+O(1)$. Is it true that for any positive constant $\varepsilon$ and any sufficiently large $p$, there exists $n \in \mathbb{Z} / p \mathbb{Z}$ satisfying $\left|R_{A}^{(1)}(n)-p / 4\right|<\varepsilon p$ ?

Problem 5 (Konyagin, personal communication) Do there exist positive constants $\varepsilon$ and $C$ such that for any sufficiently large prime $p$ and any non-empty subset $A \subseteq \mathbb{Z} / p \mathbb{Z}$ of cardinality $|A|<\sqrt{p}$, there is $n \in \mathbb{Z} / p \mathbb{Z}$ satisfying $1 \leq R_{A}^{(1)}(n) \leq C|A|^{1-\varepsilon}$ ?

\section{References}

[CW03] Y.-G. Chen and B. Wang, On the additive properties of two special sequences, Acta Arithmetica 110 (2003), no. 3, 299-303.

[D02] G. Dombi, Additive properties of certain sets, Acta Arithmetica 103 (2002), no. 2, 137-146.

[N78] M.B. Nathanson, Representation functions of sequences in additive number theory, Proc. Amer. Math. Soc. 72 (1978), no. 1, 16-20.

[P75] J.M. Pollard, Addition properties of residue classes, J. London Math. Soc. 2 (11) (1975), 147-152, 460-462. 